\title{
Long-Term Therapy with Nifedipine-CR Improves Arterio-Sclerosis Related Markers in Patients with Untreated Essential Hypertension
}

\author{
Toshihiro Kita*, Mariko Tokashiki and Kazuo Kitamura
}

\author{
Department of Internal Medicine, Division of Circulatory and Body Fluid Regulation, Faculty of Medicine, University of \\ Miyazaki, Japan
}

\begin{abstract}
Increased arteriosclerosis is associated with high risk of cardiovascular events. Several non-invasive markers for arteriosclerosis have been introduced, such as pulse wave velocity (PWV), augmentation index (AI), and carotid properties assessed by echogram, to estimate the current risk and therapeutic merit of antihypertensives. In this study, 17 hypertensive patients were treated with nifedipine-CR alone for one year, and the non-invasive markers were simultaneously monitored every 3 months. Nifedipine-CR treatment achieved stable blood pressure control, and PWV and AI improved in parallel with the blood pressure. Interestingly, the elastic property of the carotid artery progressively decreased and there was a significant difference between the results at 3 and 12 months $(85.8 \pm 6.1$ vs $72.4 \pm 5.0 \mathrm{kPa}, \mathrm{P}=0.009)$. Intima-media thickness of the carotid artery also decreased. In conclusion, nifedipine-CR demonstrated a stable anti-sclerotic quality in hypertensive patients and seems to be prominent in large arteries such as the carotid.
\end{abstract}

Keywords: Pulse wave velocity, augmentation index, elastic property, IMT, nifedipine.

\section{INTRODUCTION}

Arterial sclerosis has been recognized as an important cardiovascular risk marker. Pulse wave velocity (PWV) and augmentation index $(\mathrm{AI})$, which are themselves representative markers of arterial sclerosis, are thought to be useful indicators for anti-hypertensive therapy [1-4]. Echo assessments of the carotid artery, such as intima-media thickness (IMT) and elastic property (Ep), are other markers of arterial status. IMT reflects atherosclerotic status and is a strong predictor for cardiovascular accident [5, 6], and Ep directly represents arterial stiffness [7]. In addition, the second derivative of photoplethysmogram (SDPTG) reflects arterial aging and sclerosis [8]. While each parameter has advantages for the estimation of arterial status, no individual parameter is without its limitation, or independently sufficient. Although the administration of anti-hypertensive drugs is likely to improve some of the parameters affected during the process of arterial sclerosis, there have been very few reports on the systematic and simultaneous assessment of the effect of the drugs on these parameters. Furthermore, the multilateral assessment of arterial status may be helpful for identifying and selecting suitable antihypertensive drugs.

$\mathrm{Ca}^{2+}$ channel blockers (CCB) have been reported to possess anti-sclerotic properties in carotid and coronary arteries [9-11]. In a randomized controlled study, CCB was more effective in reducing central aortic pressure (estimated using radial AI) than $\beta$-blockers [12]. To evaluate the systematic effects of nifedipine, a representative $\mathrm{CCB}$, recently introduced non-invasive markers of arterial sclerosis, including brachial-ankle PWV (baPWV) and radial AI, and traditional

*Address correspondence to this author at the Department of Internal Medicine, Circulatory and Body Fluid Regulation, Faculty of Medicine, University of Miyazaki, 5200 Kihara, Kiyotake, Miyazaki, 889-1692, Japan;

Tel: 81-985-85-0872; Fax: 81-985-85-6596; E-mail: t-kita@po.sphere.ne.jp carotid artery scanning, and SDPTG were simultaneously monitored in patients with untreated essential hypertension. Additionally, the anti-inflammatory and anti-oxidant effects of nifedipine were assessed.

\section{MATERIALS AND METHOD}

\section{Patients}

Eighteen patients were screened and 17 with untreated essential hypertension $(55.1 \pm 8.0$ years of age, 11 men and 6 women) were enrolled in this study. All patients underwent screening examinations including urine and blood tests, electrocardiogram (ECG), chest X-ray, and echocardiography. One patient who was not enrolled was excluded because his blood pressure (BP) was 138/88 $\mathrm{mmHg}$. No patient had received prior medication for hypertension, had heart and renal failure, peripheral artery disease, or a history of cardiovascular accidents. Echocardiogram identified left ventricular hypertrophy (left ventricular mass index $>120 \mathrm{~g} / \mathrm{m}^{2}$ ) in 4 out of the 11 male patients. Two patients had hypercholesterolemia (total cholesterol $>240 \mathrm{mg} / \mathrm{dl}$ ), and one had hyperglycemia (fasting blood glucose $143 \mathrm{mg} / \mathrm{dl}$ ), however, no patient had received any prior medication for hyperlipidemia or diabetes mellitus. All 17 patients completed the study and their data were included in the analyses. The study was approved by the ethics committee of the institute, and all patients gave written informed consent.

\section{Protocol}

Seventeen patients were treated with nifedipine-controlled release (CR) (20 to $60 \mathrm{mg} /$ day) to achieve a target $\mathrm{BP}<$ $140 / 90 \mathrm{mmHg}$. The dose of nifedipine-CR was gradually increased in the first 2 to 4 weeks up to $60 \mathrm{mg} / \mathrm{day}$, to achieve the target BP. In the event that the target BP was not achieved with nifedipine-CR $60 \mathrm{mg} /$ day, no additional drugs were administered. All measurements were conducted in our outpatient office in a quiet environment and where a constant 
temperature was maintained. BP, heart rate and baPWV were measured every 3 months with an automatic waveform analyzer (form PWV/ABI, BP-203RPE; Omron Colin, Komaki, Japan) as reported previously [13]. Not only PWV but also $\mathrm{BP}$ and heart rate were measured by the automatic waveform analyzer for all data analyses. Radial AI was automatically calculated from the radial artery waveform obtained from the left wrist in the sitting position using a HEM9010AI tonometer (Omron Healthcare, Kyoto, Japan). Details of the maneuver and the principle have been well documented in a previous paper [14]. SDPTG was measured in the sitting position using an automated photoplethysmometer (DynaPulse SDP-100, Fukuda Denshi, Tokyo, Japan). Details of the maneuver and the principle have also been well documented previously [8]. We used the b/a index and d/a index of SDPTG. Takazawa et al. have suggested that the b/a index might reflect large arterial stiffness and the d/a index peripheral reflection [8]. Duplicated carotid artery scans were performed using a B-mode and M-mode ultrasonographic instrument (iE33, Philips Electronics Japan, Tokyo, Japan) with a $10 \mathrm{MHz}$ frequency scanner. Subjects were placed in the supine position and both the right and left common carotid arteries (CCA) were investigated. The CCAs were scanned comprehensively from the carotid bifurcation to the proximal portion as far as possible. Only one small plaque ( $2 \mathrm{~mm}$ in height) was found in the posterior wall of the right CCA of one patient, and this was excluded from the IMT and Ep assessments. IMT was measured at three points, one $\mathrm{cm}$ apart, on the posterior wall of CCA, starting one $\mathrm{cm}$ down from the bifurcation. The average of six points (i.e., 3 points from each side $\mathrm{X}$ of both CCAs) was taken as the IMT of the patient. Systolic and diastolic diameters of the CCAs were measured approximately $3 \mathrm{~cm}$ proximal to the bifurcation by M-mode. Ep was calculated according to the following formula [7]:

Strain $=($ Ds-Dd $) \mathrm{mm} / \mathrm{Dd} \mathrm{mm}$, Ds; systolic diameter, Dd; diastolic diameter

Ep $(\mathrm{kPa})=(\mathrm{Ps}-\mathrm{Pd}) \mathrm{mmHg} / \mathrm{strain} / 7.5 \mathrm{mmHg}$, Ps; systolic BP, $\mathrm{Pd}$; diastolic BP

BP and pulse rate were measured by automated device (HEM-907, Omron Healthcare, Kyoto, Japan). The average of four measurements (duplicated measurements of each side $\mathrm{X}$ of both CCAs) was taken as the Ep of the patient.

Blood samples were taken every 3 months and used to measure biochemical parameters and vasoactive factors; all samples were collected in the morning, in the fasting state and with the patient in the sitting position. Blood samples for the determination of adrenomedullin levels were collected in tubes containing $1 \mathrm{~g} / 1$ of EDTA and 500 kallikrein inhibition units $/ \mathrm{ml}$ of aprotinin, centrifuged immediately at $4^{\circ} \mathrm{C}$, and then stored at $-25^{\circ} \mathrm{C}$ until assayed. The plasma concentration of adrenomedullin was measured by an immunoradiometric assay kit (AM RIA Shionogi, Osaka, Japan). High sensitive C-reactive protein (hsCRP) was measured by latex nephelometry (Dade Behrling, Germany). Blood samples for the determination of the other parameters were also centrifuged immediately on collection at $4^{\circ} \mathrm{C}$ and the concentrations of these parameters were measured by a commercially available laboratory testing service (SRL, Hachioji, Japan). Urine samples from the second urination of the morning were also collected for assessments of oxidative stress markers (8-isoprostane and 8-hydroxy-2'-deoxyguanosine). These markers were also measured by the same laboratory testing service (SRL) and normalized by the creatinine concentration of the urine.

\section{Statistical Analyses}

All statistical analyses were performed using StatView-J software (version 4.5; Abacus Concepts, Inc., Berkeley, CA) on a Macintosh computer. After confirmation of normal distributions for all variables, the significance of differences was evaluated by paired $t$-test or analysis of variance (ANOVA) followed by Fisher's multiple comparison tests. Relationships between variables were analyzed by simple correlation analysis. Data are expressed as the mean \pm SEM, and a value of $\mathrm{P}<0.05$ was the criterion for statistical significance.

\section{RESULTS}

Nifedipine-CR treatment achieved stable BP control within 3 months and this was maintained until the end of the study (Fig. 1). However, heart rate was not altered by nifedipine-CR (Fig. 1).

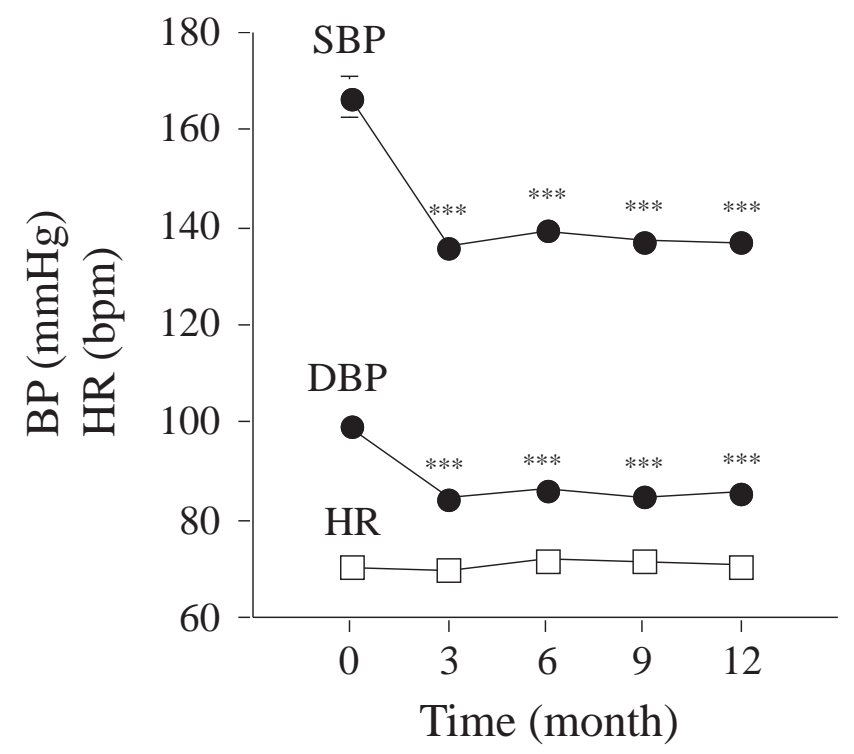

Fig. (1). Time course of systolic and diastolic blood pressure (SBP, DBP) and heart rate (HR). HR was measured by electrocardiogram (ECG). Values are mean \pm SEM, *** $\mathrm{p}<0.0001$ compared to each control value.

The average dose of nifedipine-CR at 12-month was 41.2 $\mathrm{mg} / \mathrm{day}$ ( $20 \mathrm{mg}$ for 3 patients, $40 \mathrm{mg}$ for 10 patients and 60 $\mathrm{mg}$ for 4 patients); 12 patients achieved the target BP. After 3 months, baPWV and radial AI were also decreased significantly, and these were maintained at stable levels until the end of the study (Fig. 2). Interestingly, the Ep of the carotid artery decreased progressively and there was a significant difference between the findings at 3 and 12 months $(85.8 \pm 6.1$ vs $72.4 \pm 5.0 \mathrm{kPa}, \mathrm{P}=0.009$, Fig. 2).

The IMT of the carotid artery was also decreased significantly after one-year of treatment (Fig. 3A). In addition, the $\mathrm{b} / \mathrm{a}$ index and $\mathrm{d} / \mathrm{a}$ index of SDPTG improved following treatment with nifedipine-CR (Fig. 3B). Basal conditions, such as the presence of left ventricular hypertrophy, did not 
affect the changes seen with these parameters (data not shown).

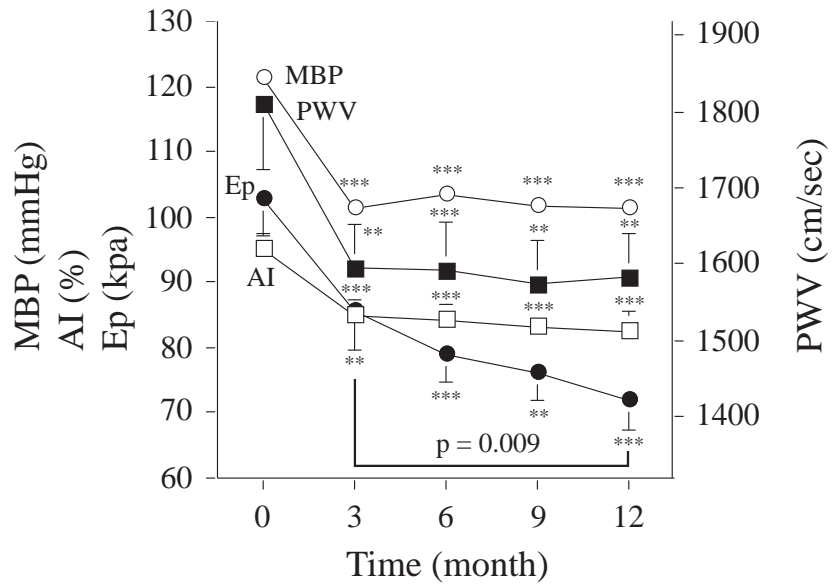

Fig. (2). Time course of mean blood pressure (MBP), pulse wave velocity (PWV), radial augmentation index (AI), and elastic property of carotid artery (Ep). Values are mean \pm SEM. $* * * p<0.0001$ and $* * \mathrm{p}<0.01$ compared to each control value.

Data concerning blood and urine samples are summarized in Table 1. Plasma noradrenalin and renin activity were significantly increased after nifedipine-CR treatment. As indicated in Table 1, other factors including inflammatory and oxidative stress markers did not change. Blood glucose and lipids including triglyceride, total cholesterol and low-density and high-density lipoprotein cholesterol also remained unchanged (data not shown).

\section{DISCUSSION}

Non-invasive and convenient devices to assess arterial sclerosis have been developed in Japan and introduced into the clinical setting. An understanding of the significance and usefulness of the markers that can be identified by the de-

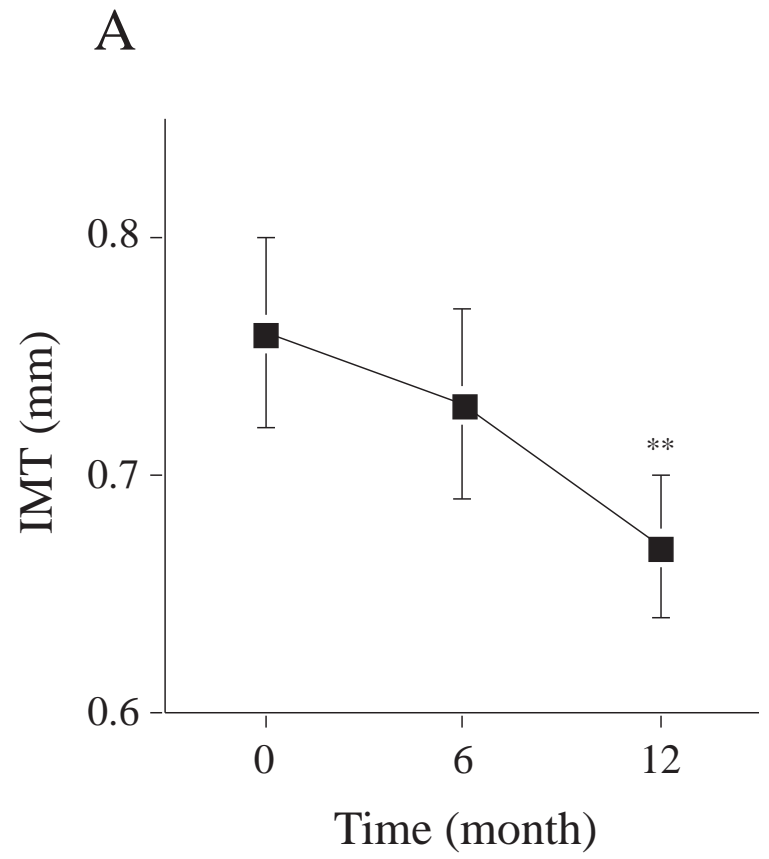

vices is accumulating for many diseases or conditions associated with arterial sclerosis. For example, baPWV is increased in patients with hypertension, diabetes mellitus, metabolic syndrome and advanced cardiovascular damages [15-18]. Furthermore, baPWV improves after antihypertensive treatment [19-20], and has been shown to be a good predictor of prognosis [21]. Radial AI has been introduced recently and although clinical data are still limited, nevertheless it has been shown that radial AI was increased in high-risk groups such as hypertensive patients with left ventricular hypertrophy [22]. On the other hand, traditional AI, which was measured on the carotid artery, is closely associated with cardiovascular diseases, especially coronary artery disease, and has a predictive power in cardiovascular accidents $[23,24]$. Echography of the carotid artery is another representative marker of arterial sclerosis. IMT of the carotid artery is a well-established marker and an IMT $>0.9 \mathrm{~mm}$ is taken as indicative of arterial damage due to hypertension, in the Guideline of ESH/ESC [25]. Carotid arterial echography also provides information of arterial stiffness or elasticity. We used the Ep of the carotid artery as a representative marker of arterial stiffness [7]. Ep indicates necessary pressure to distort definite amount of arterial wall and thus increased Ep means a stiffed artery. Also, the clinical significance of $b / a$ index and $d / a$ index of SDPTG has been reported [8].

Although these non-invasive parameters are all associated with arteriosclerosis, they do not act in concert. Furthermore, the location of the artery that has the most influence on their status, differs between the parameters. Thus, while baPWV is strongly affected by the condition of the muscular artery of the lower limbs, AI reflects the condition of the large elastic artery, and Ep is representative of the condition of the carotid artery only. These differences bring some independency to the parameters, for example, Qureshi et al. reported that IMT of the aorta was related with AI, but not with PWV [26]. This difference could be detected in this

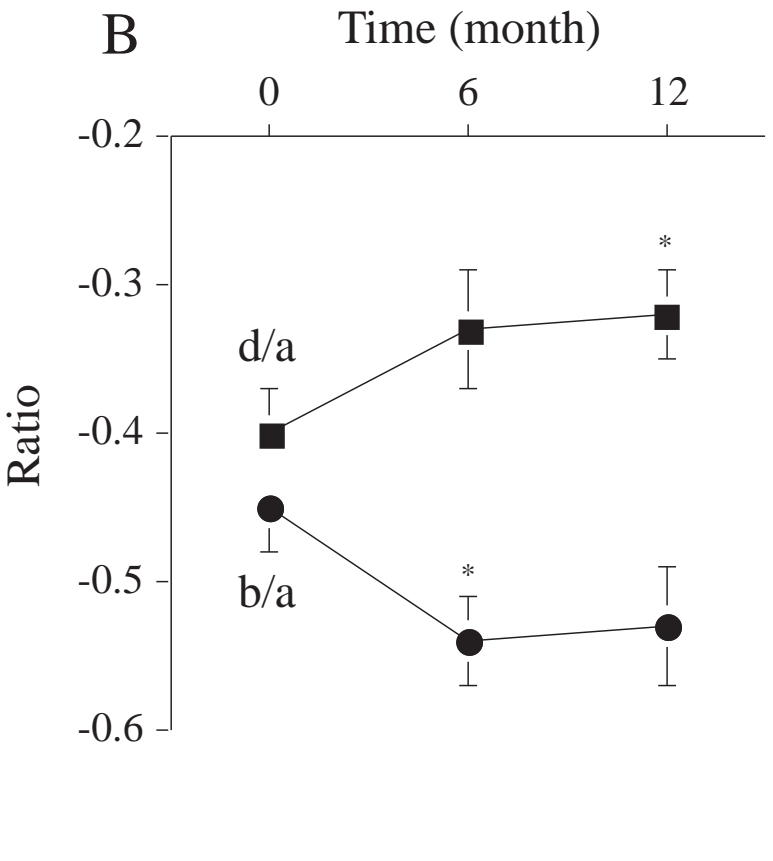

Fig. (3). Time course of intima-media thickness of carotid artery (IMT, panel A). Time course of b/a index and d/a index of second derivative of photoplethysmogram (panel $\mathbf{B}$ ). Values are mean \pm SEM, $* \mathrm{p}<0.05$ compared to each control value. 
Table 1. Time-Dependent Effects of Nifedipine-CR on Indicated Parameters

\begin{tabular}{|l|c|c|c|c|c|}
\hline \multirow{2}{*}{} & \multirow{2}{*}{ Control } & \multicolumn{3}{|c|}{ Treatment with Nifedipine-CR } \\
\cline { 3 - 6 } & & 3 Months & 6 Months & 9 Months & 12 Months \\
\hline \hline IRI (mU/ml) & $9.1 \pm 0.9$ & $8.5 \pm 0.8$ & $10.3 \pm 1.2$ & $8.4 \pm 0.7$ & $9.8 \pm 1.0$ \\
\hline hs-CRP (ng/ml) & $1060 \pm 415$ & $866 \pm 302$ & $1078 \pm 297$ & $1038 \pm 312$ & $1087 \pm 320$ \\
\hline PRA (ng/ml/hr) & $0.9 \pm 0.2$ & $1.7 \pm 0.3^{*}$ & $1.8 \pm 0.4^{*}$ & $1.7 \pm 0.4^{*}$ & $1.8 \pm 0.5^{*}$ \\
\hline PAC (pg/ml) & $102 \pm 10$ & $118 \pm 13$ & $105 \pm 10$ & $107 \pm 11$ & $104 \pm 12$ \\
\hline Adrenalin (pg/ml) & $59.7 \pm 6.4$ & $47.2 \pm 4.7$ & $42.1 \pm 5.2$ & $49.3 \pm 6.0$ & $41.1 \pm 3.9$ \\
\hline Noradrenalin (pg/ml) & $517 \pm 29$ & $682 \pm 59^{*}$ & $659 \pm 61$ & $644 \pm 61^{*}$ & $654 \pm 60^{*}$ \\
\hline Adrenomedullin (fmol/ml) & $11.2 \pm 1.0$ & $11.6 \pm 0.8$ & $14.0 \pm 1.0$ & $13.3 \pm 0.6$ & $13.9 \pm 0.7$ \\
\hline MDA-LDL (U/l) & $138 \pm 16$ & $150 \pm 13$ & $146 \pm 15$ & $131 \pm 14$ & $151 \pm 19$ \\
\hline 8-Isoprostane (pg/mg Cre) & $215 \pm 21$ & $237 \pm 61$ & $227 \pm 32$ & $258 \pm 61$ & $287 \pm 96$ \\
\hline 8-OHdG (pg/mg Cre) & $9.0 \pm 1.0$ & $10.5 \pm 1.1$ & $14.9 \pm 3.5$ & $10.2 \pm 0.9$ & $12.8 \pm 1.5$ \\
\hline
\end{tabular}

IRI: Immunoreactive insulin, hs-CRP: high sensitive C-reactive protein.

PRA: Plasma renin activity, PAC: plasma aldosterone concentration.

MDA-LDL: Malondialdehyde-low density lipoprotein cholesterol.

8-OHdG: 8-Hydroxy-2'-deoxyguanosine.

$* \mathrm{P}<0.05$, compared to control.

study; the reduction of AI correlated strongly with the change of carotid IMT, but PWV did not (Fig. 4). Also, there is a different relationship between organ damage and these parameters, e.g. previous reports have shown that diabetic retinopathy was associated with PWV but not with AI [27].
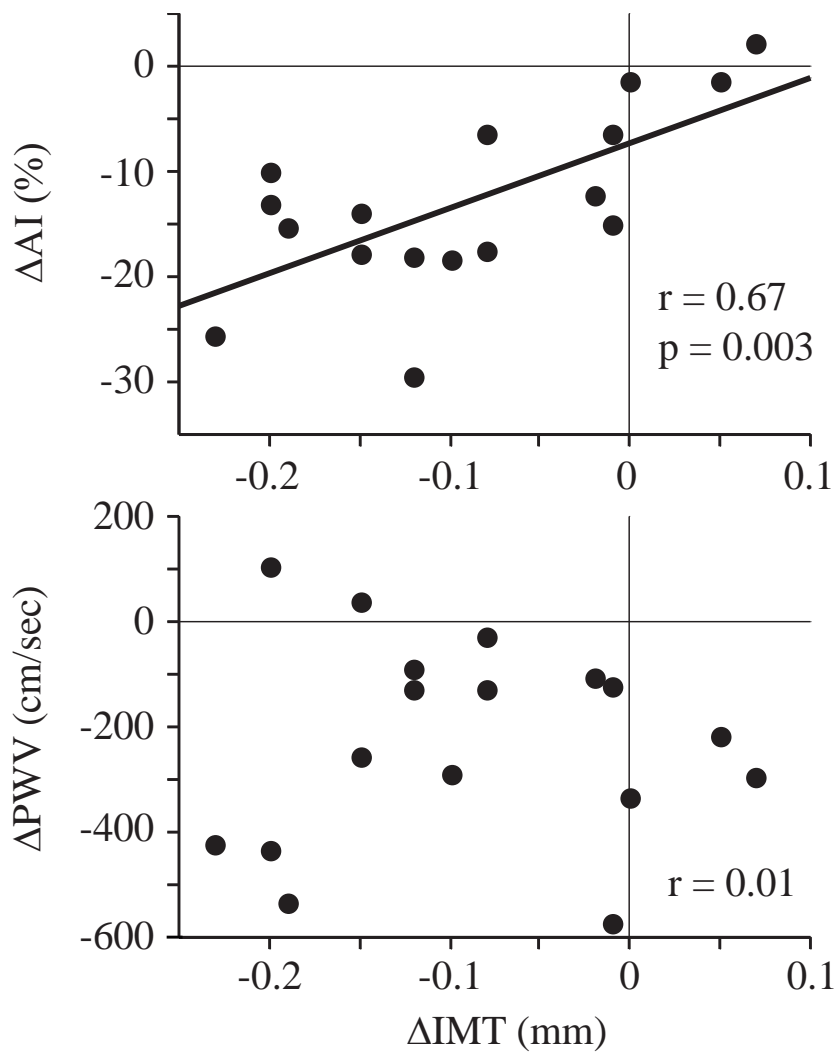

Fig. (4). The relationship between decreases of augmentation index $(\triangle \mathrm{AI})$ and decreases of intima-media thickness of carotid artery $(\triangle \mathrm{IMT})$ in the upper panel, and the relationship between decreases of pulse wave velocity $(\Delta \mathrm{PWV})$ and $\Delta \mathrm{IMT}$ in the lower panel.
$\mathrm{AI}$ in particular seems to be independent of the other parameters, and this observation led Sakurai et al. to propose that AI may not be a true indicator of arterial stiffness, but an index of wave reflection including PWV [28]. There is also a difference in the response of the parameters to antihypertensive drugs; thus, in antihypertensive patients, while both beta-blockers and an ACE inhibitor/diuretic combination decreased PWV, only the ACE inhibitor/diuretic combination improved AI [2]. Additionally, there is a degree of independence between blood pressure reduction by anti-hypertensive drugs and improvement of PWV [29]. These findings suggest that the multilateral assessment of arterial status may be helpful for evaluating antihypertensive drugs.

In our study, all markers of arterial stiffness and sclerosis were improved following nifedipine-CR treatment (Figs. 2, 3). Systematic and simultaneous assessment of the markers clearly showed the anti-sclerotic ability of nifedipine-CR. Simultaneous assessment of several markers can compensate for the weak spots of the individual markers. For example, baPWV is highly BP dependent, while radial AI is not completely independent from BP $[14,20]$; on the other hand, IMT of the carotid artery is not affected by present BP. Heart rate has a direct effect on radial AI [14] and little on baPWV [30], fortunately, however, average heart rate was unchanged throughout the study (Fig. 1). The association between parameters, such as baPWV and radial AI, is relatively poor [28]. Even though the number of patients in our study was relatively small, we demonstrated quite weak correlations among baPWV, radial AI, carotid IMT and carotid Ep, with the exception of IMT and Ep $(r=0.54, \mathrm{P}=0.025)$ at baseline. Therefore, the parallel decrease of baPWV and radial AI could be a strong indication of steady improvement of the arterial sclerosis.

Interestingly, Ep of the carotid artery was progressively improved after 3 months of nifedipine-CR treatment. As is obvious from the equation, Ep is influenced by BP. However, as BP remained stable after 3 months (Fig. 1), its affect 
after this time point can be regarded as minimal. Thus it is strongly suggested that this progressive improvement of Ep was caused by organic improvement of the CCA. Additionally, the progressive decrease of IMT suggested beneficial organic alteration of the carotid artery (Fig. 3A); certainly IMT has no immediate connection with Ep of the carotid artery. This observation indicates that the effect of nifedipine-CR is prominent in the carotid artery, and an improved performance might contribute to cerebrovascular protection that is of proven benefit in CCB [31].

Contrary to our expectation and previous reports [32, 33], nifedipine-CR had a neutral effect on the inflammatory and oxidative stress markers evaluated in this study (Table 1). Also, nifedipine-CR did not affect the plasma concentration of adrenomedullin, as previously observed with benidipine [20]. On the other hand, nifedipine-CR obviously stimulated renin and sympathetic nerve activities (Table 1), probably caused by reflex sympathetic stimulation of the drug [34, 35]. This renin and sympathetic nerve activation may cancel any favorable effects that nifedipine-CR has against inflammatory and oxidative stress markers. Additionally, sympathetic nerve activation could decrease the improvement of baPWV. A similar phenomenon was reported by Munakata et al. [36]; a relatively small improvement of baPWV following nifedipine treatment was associated with increased plasma noradrenalin in hypertensive patients. The discrepancy in the fall in Ep and baPWV after nifedipine-CR treatment, namely the non-progressive decrease of baPWV, may be caused, partially, by this renin and sympathetic nerve activation. Additional combination therapy to nifedipine-CR treatment to inhibit renin-angiotensin system and/or sympathetic nerve system may provide a more favorable outcome for arterial sclerosis.

The potential limitations of this study merit consideration. First, there was no control group who was treated by other antihypertensive drugs or other interventions, eg strict dietary salt intake restrictions. This made it hard to separate the unique merit of nifedipine-CR against arteriosclerosis from the effect of the accompanying lowering of the blood pressure. Although a multi-treatment arm study may clarify the relative merits of each drug, it is almost impossible to extract pure merit of antihypertensives beyond blood pressure lowering. At least in our present study, nifedipine-CR improved an entire range of arteriosclerosis-related-markers that were capable of responding independently of each other (example in Fig. 4). Therefore it appears that it is highly probable that this drug can preferentially modify arteriosclerosis. Second, prolonged elevation of catecholamine levels may interfere with the initial merit of nifedipine-CR, for example IMT regression. Longer term observation may be helpful in clarifying this possibility. Third, the number of patients in this study was small, and thus the result must be confirmed in a larger number of participants in a separate study. Furthermore, it is hard to verify completely the inter-relationship or independence of each parameter with this small number of patients.

In conclusion, nifedipine-CR demonstrated a stable anti-sclerotic property in hypertensive patients and it seems to be prominent in the carotid artery. The steady hypotensive effect and anti-sclerotic property of nifedipine-CR should be beneficial for the prevention of cardiovascular accidents in hypertensive patients.

\section{REFERENCES}

[1] Laurent S, Boutouyrie P, Asmar R, et al. Aortic stiffness is an independent predictor of all-cause and cardiovascular mortality in hypertensive patients. Hypertension 2001; 37: 1236-41.

[2] Asmar RG, London GM, O'Rourke ME, Safar ME, REASON Project Coordinators and Investigators. Improvement in blood pressure, arterial stiffness and wave reflections with a very-low-dose perindopril/indapamide combination in hypertensive patient: a comparison with atenolol. Hypertension 2001; 38: 922-6.

[3] Guerin AP, Blacher J, Pannier B, Marchais SJ, Safar ME, London GM. Impact of aortic stiffness attenuation on survival of patients in end-stage renal failure. Circulation 2001; 103: 987-92.

[4] Benetos A, Adamopoulos C, Bureau J-M, et al. Determinants of accelerated progression of arterial stiffness in normotensive subjects and in treated hypertensive subjects over a 6-year period. Circulation 2002; 105: 1202-7.

[5] Touboul PJ, Labreuche J, Vicaut E, Amarenco P, GENIC Investigators. Carotid intima-media thickness, plaques, and Framingham risk score as independent determinants of stroke risk. Stroke 2005; 36: 1741-5.

[6] Lorenz MW, von Kegler S, Steinmetz H, Markus HS, Sitzer M. Carotid intima-media thickening indicates a higher vascular risk across a wide age range: prospective data from the Carotid Atherosclerosis Progression Study (CAPS). Stroke 2006; 37: 87-92.

[7] Blankenhorn DH, Chin HP, Conover DJ, Nessim SA. Ultrasound observation on pulsation in human carotid artery lesions. Ultrasound Med Biol 1988; 14: 583-7.

[8] Takazawa K, Tanaka N, Fujita M, et al. Assessment of vasoactive agents and vascular aging by the second derivative of photoplethysmogram waveform. Hypertension 1998; 32: 365-70.

[9] Pitt B, Byington RP, Furberg CD, et al. Effect of amlodipine on the progression of atherosclerosis and the occurrence of clinical events. PREVENT Investigators. Circulation 2000; 102: 1503-10.

[10] Zanchetti A, Bond MG, Henning M, et al. Calcium antagonist lacidipine slows down progression of asymptomatic carotid atherosclerosis: principal results of the European Lacidipine Study on Atherosclerosis (ELSA), a randomized, double-blind, long-term trial. Circulation 2002; 106: 2422-7.

[11] Nissen SE, Tuzcu EM, Libby P, et al. Effect of antihypertensive agents on cardiovascular events in patients with coronary disease and normal blood pressure: the CAMELOT study: a randomized controlled trial. JAMA 2004; 292: 2217-25.

[12] Williams B, Lacy PS, Thom SM, et al. Differential impact of blood pressure-lowering drugs on central aortic pressure and clinical outcomes: principal results of the Conduit Artery Function Evaluation (CAFE) study. Circulation 2006; 113: 1213-25.

[13] Kita T, Kitamura K, Hashida S, Morishita K, Eto T. Plasma adrenomedullin is closely correlated with pulse wave velocity in middle-aged and elderly patients. Hypertens Res 2003; 26: 887-93.

[14] Kohara K, Tabara Y, Oshiumi A, Miyawaki Y, Kobayashi T, Miki T. Radial augmentation index: a useful and easily obtainable parameter for vascular aging. Am J Hypertens 2005; 18: 11S-14S.

[15] Yamashina A, Tomiyama H, Arai T, et al. Brachial-ankle pulse wave velocity as a marker of atherosclerotic vascular damage and cardiovascular risk. Hypertens Res 2003; 26: 615-22.

[16] Aso K, Miyata M, Kubo T, et al. Brachial-ankle pulse wave velocity is useful for evaluation of complications in type 2 diabetic patients. Hypertens Res 2003; 26: 807-13.

[17] Saijo Y, Yoshioka E, Fukui T, Kawaharada M, Kishi R. Metabolic syndrome, C-reactive protein and increased arterial stiffness in Japanese subjects. Hypertens Res 2006; 29: 589-96.

[18] Matsumoto M, Inoue K, Moriki A. Associations of brachial-ankle pulse wave velocity and carotid atherosclerotic lesions with silent cerebral lesions. Hypertens Res 2007; 30: 767-73.

[19] Ichihara A, Hayashi M, Koura Y, Tada Y, Hirota N, Saruta T. Long-term effects of intensive blood pressure lowering on arterial wall stiffness in hypertensive patients. Am J Hypertens 2003; 16: 959-65.

[20] Kita T, Suzuki Y, Eto T, Kitamura K. Long-term anti-hypertensive therapy with benidipine improves arterial stiffness over blood pressure lowering. Hypertens Res 2005; 28: 959-64. 
[21] Kitahara T, Ono K, Tsuchida A, et al. Impact of brachial-ankle pulse wave velocity and ankle-brachial blood pressure index on mortality in hemodialysis patients. Am J Kidney Dis 2005; 46: 688-96.

[22] Hashimoto J, Watabe D, Hatanaka R, et al. Enhanced radial late systolic pressure augmentation in hypertensive patients with left ventricular hypertrophy. Am J Hypertens 2006; 19: 27-32.

[23] Weber T, Auer J, O'Rourke MF, et al. Arterial stiffness, wave reflections, and the risk of coronary artery disease. Circulation 2004; 109: 184-9.

[24] Weber T, Auer J, O'Rourke MF, et al. Increased arterial wave reflections predict severe cardiovascular events in patients undergoing percutaneous coronary interventions. Eur Heart J 2005; 26: 2657-63.

[25] Mancia G, De Backer G, Dominiczak A, et al. 2007 Guidelines for the Management of Arterial Hypertension: The Task Force for the Management of Arterial Hypertension of the European Society of Hypertension (ESH) and of the European Society of Cardiology (ESC). J Hypertens 2007; 25: 1105-87.

[26] Quershi G, Brown R, Salciccioli L, et al. Relationship between aortic atherosclerosis and non-invasive measures of arterial stiffness. Atherosclerosis 2007; 195: e190-e4.

[27] Ogawa O, Hiraoka K, Watanabe T, et al. Diabetic retinopathy is associated with pulse wave velocity, not with the augmentation index of pulse waveform. Cardiovasc Diabetol 2008; 7: 11.

[28] Sakurai M, Yamakado T, Kurachi H, et al. The relationship between aortic augmentation index and pulse wave velocity; an invasive study. J Hypertens 2007; 25: 391-7.
[29] Asmar R. Effect of antihypertensive agents on arterial stiffness as evaluated by pulse wave velocity; clinical implications. Am J Cardiovasc Drugs 2001; 1: 387-97.

[30] Su HM, Lee KT, Chu CS, et al. Effects of heart rate on brachial-ankle pulse wave velocity and ankle-brachial pressure index in patients without significant organic heart disease. Angiology 2007; 58: 67-74.

[31] Bangalore S, Messerli FH. A review of stroke in patients with hypertension and coronary artery disease: Focus on calcium channel blockers. Int J Clin Pract 2006; 60: 1281-6.

[32] Yamagishi S, Nakamura K, Takenaka K, Matsui T, Inoue H. Pleiotropic effects of nifedipine on atherosclerosis. Curr Pharm Des 2006; 12: 1543-7.

[33] Agabiti Rosei E, Morelli P, Rizzoni D. Effects of nifedipine GITS $20 \mathrm{mg}$ or enalapril $20 \mathrm{mg}$ on blood pressure and inflammatory markers in patients with mild-moderate hypertension. Blood Pressure 2005; 1 (Suppl): 14-22.

[34] Lederballe Pedersen O. Calcium blockade in arterial hypertension. Review. Hypertension 1983; 5: 1174-9.

[35] Wenzel RR, Allegranza G, Binggeli C, et al. Differential activation of cardiac and peripheral sympathetic nervous system by nifedipine: role of pharmacokinetics. J Am Coll Cardiol 1997; 29: 1607-14.

[36] Munakata M, Nagasaki A, Nunokawa T, et al. Effects of valsartan and nifedipine coat-core on systemic arterial stiffness in hypertensive patients. Am J Hypertens 2004; 17: 1050-5.

This is an open access article licensed under the terms of the Creative Commons Attribution Non-Commercial License (http: //creativecommons.org/licenses/by-nc/ 3.0/) which permits unrestricted, non-commercial use, distribution and reproduction in any medium, provided the work is properly cited. 